\title{
Caltsacoryne setouchiensis (Hydrozoa, Anthoathecata) a new genus and species of hydrozoan jellyfish from Japan
}

\author{
SHO TOSHINO ${ }^{*}$, YOSHIMI HAMATSU ${ }^{2} \&$ HIROAKI UCHIDA $^{2}$ \\ ${ }^{1}$ Kuroshio Biological Research Foundation, 560 Nishidomari, Otsuki, Kochi 788-0333, Japan \\ ${ }^{2}$ Nagisa Aquarium, 22111-3 Ihota, Suo-Oshima, Yamaguchi 742-2601, Japan \\ *Corresponding author. $="$ toshino@kuroshio.or.jp
}

\begin{abstract}
We report a new genus and species of hydrozoan jellyfish belonging to the order Anthoathecata collected from the Seto Inland Sea, western Japan. Caltsacoryne setouchiensis gen. et sp. n. can be distinguished from other species of Corynidae based on the following combination of morphological characters: number of tentacles, cnidocyst pads, manubrium length, and the shape of the gonad and tentacles. A table comparing the primary diagnostic characters of this new genus of Corynidae is presented.
\end{abstract}

Key words: Anthomedusae, Corynidae, hydroid, medusa, Seto Inland Sea

\section{Introduction}

The hydrozoan family Corynidae Johnston, 1836 contains approximately 50 species in 13 genera (Nawrocki et al. 2009). Species of Corynidae are widely distributed in the shallow waters of the Atlantic, Pacific, and Indian oceans (Mayer 1910; Kramp 1959), a majority of which are characterized by an alternation of generations, with life cycles comprising asexual benthic hydroids and sexual planktonic medusae (Petersen 1990; Brinckmann-Voss 2000). Typically, free-swimming medusae are liberated by the budding of hydroids, although some corynids lack medusae and produce fixed gonophores (Schuchert 2001).

Historically, the taxonomy of the family Corynidae has been problematic due to the limited differences in morphological characters among genera. Johnston (1836) erected the family Corynidae, the first genus of which was Coryne Gaertner 1774. Coryne muscoides (Linnaeus, 1761), originally described as Tubularia muscoides Linnaeus, 1761, was the first identified species. Until the 1970s, the genera, Coryne, Sarsia Lesson, 1843, and Dipurena McCrady, 1859, were the major corynid taxa (Millard 1975). Bouillon (1985) later incorporated three pre-existing genera into the family, namely, Bibrachium Stechow, 1919, Dicodonium Haeckel, 1879, and Sarsiella Lesson, 1843. However, subsequent morphological analyses failed to establish any convincing synapomorphies for the family Corynidae, thereby indicating that it may be polyphyletic (Schuchert 2001). Recent molecular phylogenetic studies lend support to this hypothesis and furthermore provide evidence to indicate that the three major corynid genera Sarsia, Dipurena, and Coryne are polyphyletic (Collins et al. 2005; Nawrocki et al. 2010). Moreover, Nawrocki et al. (2010) placed the family Polyorchidae, which includes Polyorchis A. Agassiz, 1862 and Scrippsia Torrey, 1909, among the genera of Corynidae.

To date, eight species of Corynidae have been reported from Japanese waters (Table 1), and in present study, we collected seven specimens of a hitherto unidentified species of Corynidae from the Seto Inland Sea, western Japan. On the basis our morphological and molecular phylogenetic analyses of these specimens, we propose that this species of Anthoathecata should be recognized as a newly erected genus and a new species within the family Corynidae. 
TABLE 1. List of Japanese Corynidae: a, Kubota 2014; b, Kubota \& Gravili 2007.

\begin{tabular}{lll}
\hline Scientific name & Japanese name & Reference \\
\hline Cladosarsia capitata Bouillon, 1978 & Edawakare-sarusia-kurage & $\mathrm{a}$ \\
Coryne polyocellata (Uchida, 1927) & Itsutsume-sarusia-kurage & $\mathrm{b}$ \\
Polyorchis karafutoensis Kishinouye, 1910 & Kitakami-kurage & $\mathrm{b}$ \\
Sarsia tubulosa (M. Sars, 1835) & Sarusia-kurage & $\mathrm{b}$ \\
Sarsia princeps (Haeckel, 1879) & Osarusia-kurage & $\mathrm{b}$ \\
Spirocodon saltatrix (Tilesius, 1818) & Kami-kurage & $\mathrm{b}$ \\
Stauridiosarsia ophiogaster (Haeckel, 1879) & Jyuzu-kurage & $\mathrm{a}, \mathrm{b}$ \\
Stauridiosarsia nipponica (Uchida, 1927) & Yamato-sarusia-kurage & $\mathrm{a}$ \\
\hline
\end{tabular}

\section{Methods}

Collection and fixing. Specimens were collected from Okikamuro Island, Suo-Oshima, Yamaguchi Prefecture, western Japan, between April 24, 2016 and April 18, 2018 (Fig. 1). The seven medusae were taken using a scoop (170 $\mathrm{mm}$ in diameter), among which, five individuals were fixed in $5 \%$ formalin in seawater and subsequently deposited at the National Museum of Nature and Science, Tsukuba, Japan (NSMT), whereas the remaining two specimens were preserved in $99.5 \%$ ethanol for DNA extraction.

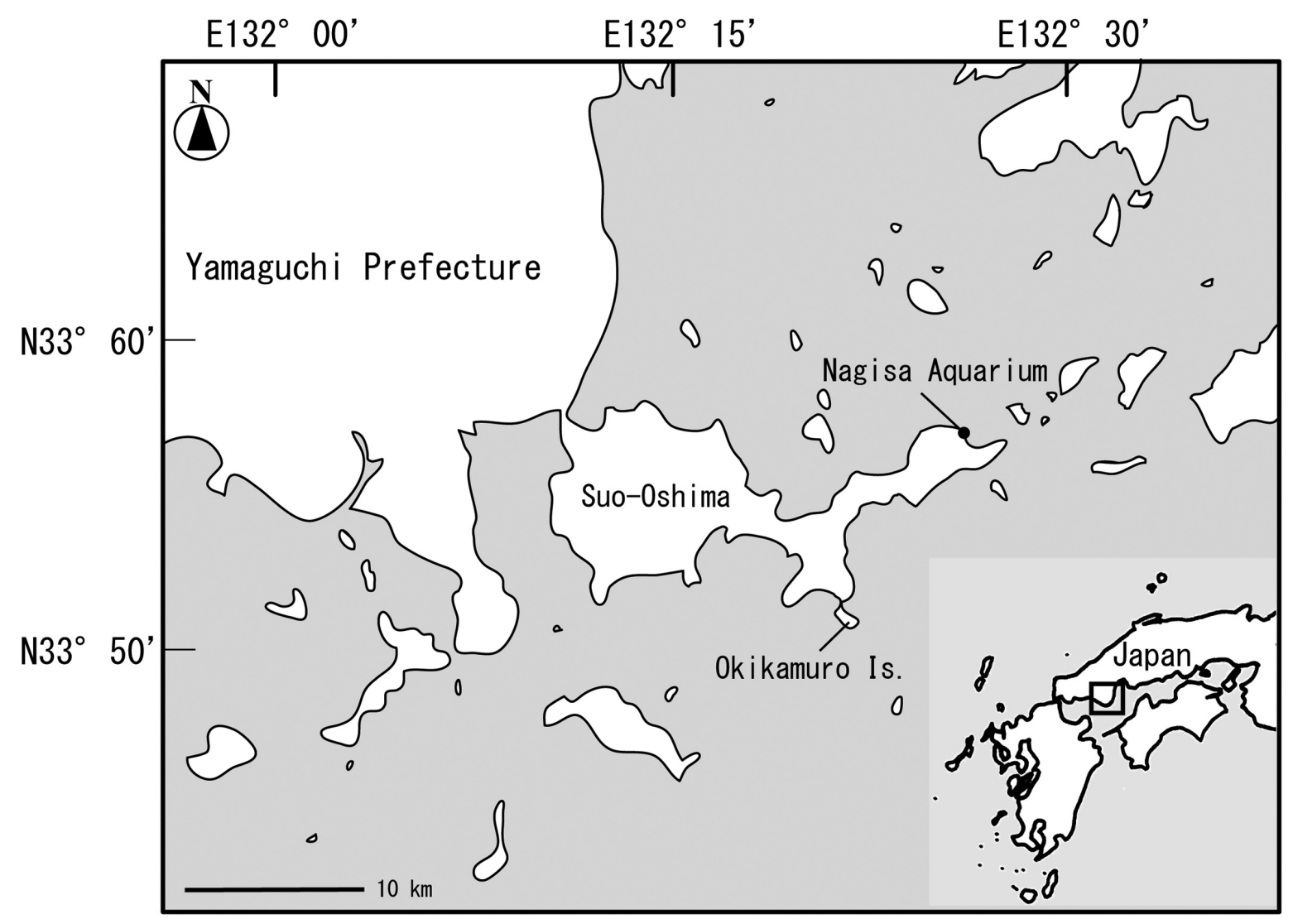

FIGURE 1. Map of the sampling sites.

Molecular phylogenetic analysis. For the purposes of molecular phylogenetic analysis, we used near complete sequences of $16 \mathrm{~S}$ rDNA. Genomic DNA was extracted from the $99.5 \%$ ethanol-preserved tissue of two specimens using a DNeasy Blood and Tissue Kit (Qiagen, Germany) following the manufacturer's protocol. Mitochondrial 16S rDNA (approximately $600 \mathrm{bp}$ ) was PCR amplified and sequenced with the primer pair fwd: TCGACTGTTTACCAAAAACATAGC and rev: ACGGAATGAACTCAAATCATGTAAG (Cunningham \& Buss 1993), using the following PCR profile: an initial denaturation at $94^{\circ} \mathrm{C} / 5 \mathrm{~min}$; five cycles of $94^{\circ} \mathrm{C} / 50 \mathrm{~s}, 45^{\circ} \mathrm{C} / 50 \mathrm{~s}$, and $72^{\circ} \mathrm{C} / 60$ 
s; 30 cycles of $94^{\circ} \mathrm{C} / 50 \mathrm{~s}, 50^{\circ} \mathrm{C} / 50 \mathrm{~s}$, and $72^{\circ} \mathrm{C} / 60 \mathrm{~s}$; and a final elongation at $72^{\circ} \mathrm{C} / 5 \mathrm{~min}$ (Collins et al. 2008). PCR products were purified using a QIAquick PCR Purification Kit (Qiagen, Germany) and sequenced in both directions using an ABI 3730 automated sequencer (Applied Biosystems). The sequences thus obtained were aligned using MEGA 6 with built-in ClustalW (Tamura et al. 2013). Phylogenetic analysis and pairwise distance measurements were performed using the maximum likelihood method based on the Kimura 2-parameter model (Kimura 1980) with 1000 bootstrap replications in MEGA 6. Two sequences have been deposited in GenBank under accession numbers LC635086 and LC635087 for the new genus (Table 2).

TABLE 2. Taxa included in the phylogenetic analyses and GenBank accession numbers for sequences: Sequences obtain ed in this study are in bold.

\begin{tabular}{|c|c|c|c|}
\hline Family & Species & Accessioned name & Accession No. \\
\hline Corynidae & Caltsacoryne setouchiensis n. sp. & Caltsacoryne setouchiensis n. sp. & LC635086 \\
\hline Corynidae & Caltsacoryne setouchiensis $\mathbf{n}$. sp. & Caltsacoryne setouchiensis $\mathbf{n}$. sp. & LC635087 \\
\hline Corynidae & Codonium prolifera & Coryne prolifera & GQ395318 \\
\hline Corynidae & Stauridiosarsia cliffordi & Coryne cliffordi & GQ395313 \\
\hline Corynidae & Coryne epizoica & Coryne epizoica & GQ395314 \\
\hline Corynidae & Coryne epizoica & Coryne epizoica & KX355418 \\
\hline Corynidae & Coryne eximia & Coryne eximia & AJ878712 \\
\hline Corynidae & Coryne eximia & Coryne eximia & KM402029 \\
\hline Corynidae & Coryne fucicola & Coryne fucicola & AM084259 \\
\hline Corynidae & Coryne muscoides & Coryne muscoides & EU876546 \\
\hline Corynidae & Coryne muscoides & Coryne muscoides & GQ395315 \\
\hline Corynidae & Coryne pintneri & Coryne pintneri & AJ878717 \\
\hline Corynidae & Coryne pintneri & Coryne pintneri & AJ878718 \\
\hline Corynidae & Coryne pusilla & Coryne pusilla & KP776759 \\
\hline Corynidae & Coryne pusilla & Coryne pusilla & KP776762 \\
\hline Corynidae & Coryne uchidai & Coryne uchidai & GQ395320 \\
\hline Corynidae & Coryne uchidai & Coryne uchidai & KP776809 \\
\hline Corynidae & Polyorchis penicillatus & Polyorchis penicillatus & KX355411 \\
\hline Corynidae & Polyorchis penicillatus & Polyorchis penicillatus & KX355412 \\
\hline Corynidae & Sarsia apicula & Sarsia apicula & GQ395330 \\
\hline Corynidae & Sarsia lovenii & Sarsia lovenii & AJ608796 \\
\hline Corynidae & Sarsia lovenii & Sarsia lovenii & AY787876 \\
\hline Corynidae & Sarsia striata & Sarsia striata & GQ395328 \\
\hline Corynidae & Sarsia striata & Sarsia striata & KX355408 \\
\hline Corynidae & Sarsia tubulosa & Sarsia tubulosa & AJ878720 \\
\hline Corynidae & Sarsia tubulosa & Sarsia tubulosa & AY512545 \\
\hline Corynidae & Scrippsia pacifica & Scrippsia pacifica & KX355419 \\
\hline Corynidae & Slabberia halterata & Dipurena halterata & AM084261 \\
\hline Corynidae & Stauridiosarsia cliffordi & Stauridiosarsia cliffordi & GQ395313 \\
\hline Corynidae & Stauridiosarsia gemmifera & Dipurena gemmifera & EU876547 \\
\hline Corynidae & Stauridiosarsia marii & Stauridiosarsia marii & AY512544 \\
\hline Corynidae & Stauridiosarsia nipponica & Coryne nipponica & GQ395316 \\
\hline Corynidae & Stauridiosarsia nipponica & Coryne nipponica & GQ395333 \\
\hline Corynidae & Stauridiosarsia ophiogaster & Dipurena ophiogaster & AJ878721 \\
\hline Corynidae & Stauridiosarsia producta & Coryne producta & AY512543 \\
\hline Corynidae & Stauridiosarsia producta & Coryne producta & GQ395317 \\
\hline Corynidae & Stauridiosarsia reesi & Dipurena reesi & GQ395321 \\
\hline Corynidae & Stauridiosarsia sp. & Dipurena sp. & GQ395331 \\
\hline Cladonematidae & Cladonema radiatum & Cladonema radiatum & AM088482 \\
\hline
\end{tabular}


Morphological analyses. Taxonomic observations and measurements were performed using living or preserved specimens (Fig. 2). Measurements to the nearest $0.1 \mathrm{~mm}$ were made using ImageJ software (Rasband 2021). Nematocysts in the medusae were identified according to Kubota (1991) and Östman (2000) using squashes prepared from fresh tissues examined under a BX 53 compound microscope (Olympus, Japan). To determine the abundance of different nematocyst types, we examined approximately 300 nematocysts from cultured specimens, which were identified, measured, and counted.

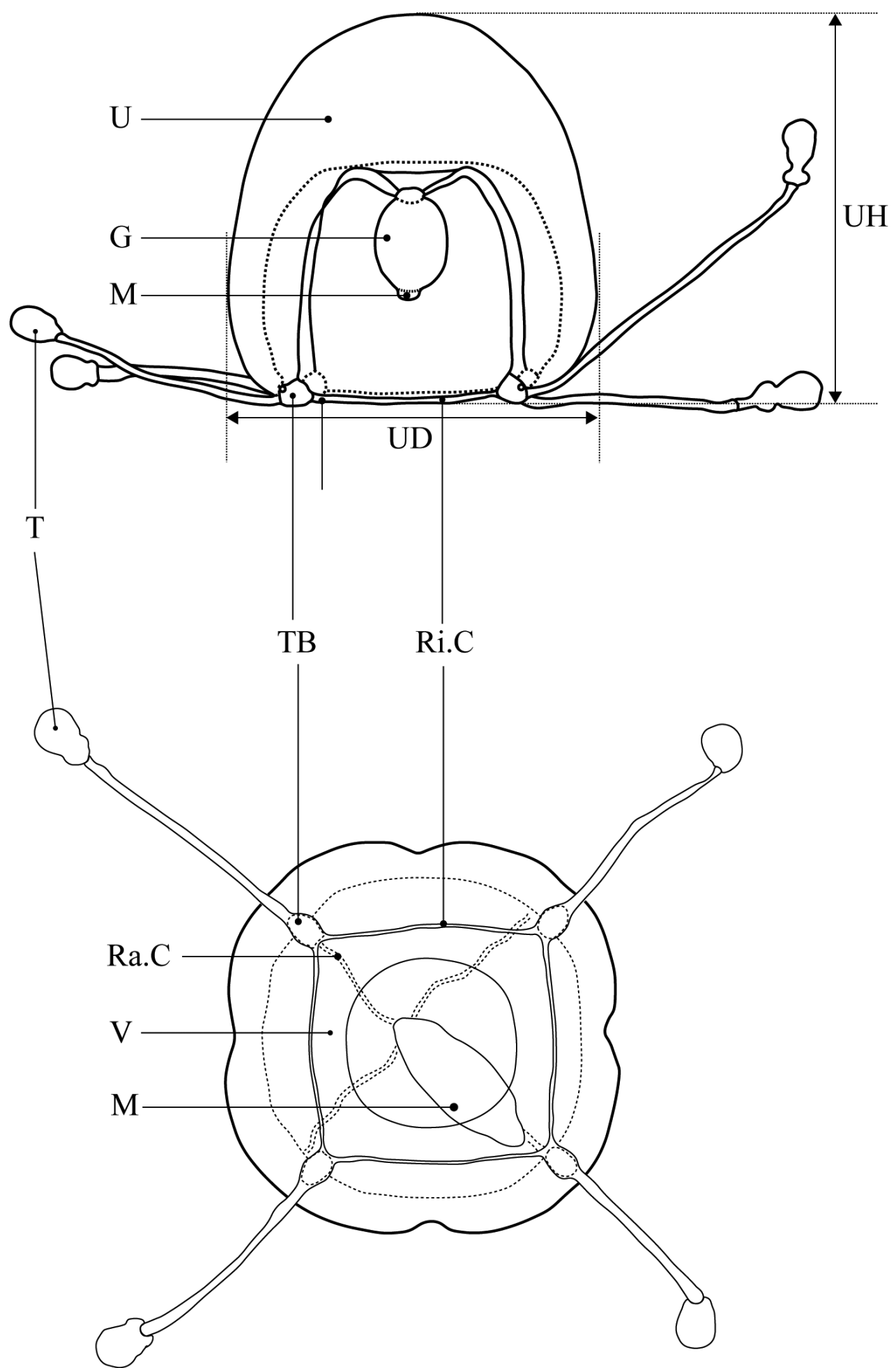

FIGURE 2. Key characters for identification and measurement of parts of the Corynidae. $G=$ gonad; $M=$ manubrium; Ra.C = radial canal; Ri.C = ring canal; $\mathrm{T}=$ tentacle; $\mathrm{TB}=$ tentacle bulb; $\mathrm{U}=$ umbrella; $\mathrm{UD}=$ umbrella diameter; $\mathrm{UH}=$ umbrella height; $\mathrm{V}=$ velum 


\section{Results}

Molecular phylogenetics. The maximum likelihood tree constructed for the family Corynidae based on 16S rDNA sequences (Fig. 3) was found to comprise two major clades, one of which contained Codonium, Coryne, Scrippsia, Polyorchis, the unidentified Corynidae, Slabberia, and Sarsia, whereas the other comprised the single genus Stauridiosarsia. The monophyly of the unidentified Corynidae from Japan was evident in the 16S phylogenetic tree (as indicated by the high bootstrap value of 92\%), thereby supporting the validity of designating this taxon as a new genus. The Kimura 2-parameter distances between the unidentified Corynidae and other genera ranged from 0.06 to 0.18 (Table 3). The K2P distance factor between Sarsia and Slabberia was found to be comparatively low, ranging from 0.03 to 0.04, whereas intergeneric distances for Sarsia, Stauridiosarsia, and Coryne were 0.01-0.02, 0.05-0.16, and 0.01-0.19, respectively. Accordingly, the K2P divergence factor of between 0.06 and 0.19 could be considered as a threshold for discriminating corynid genera.

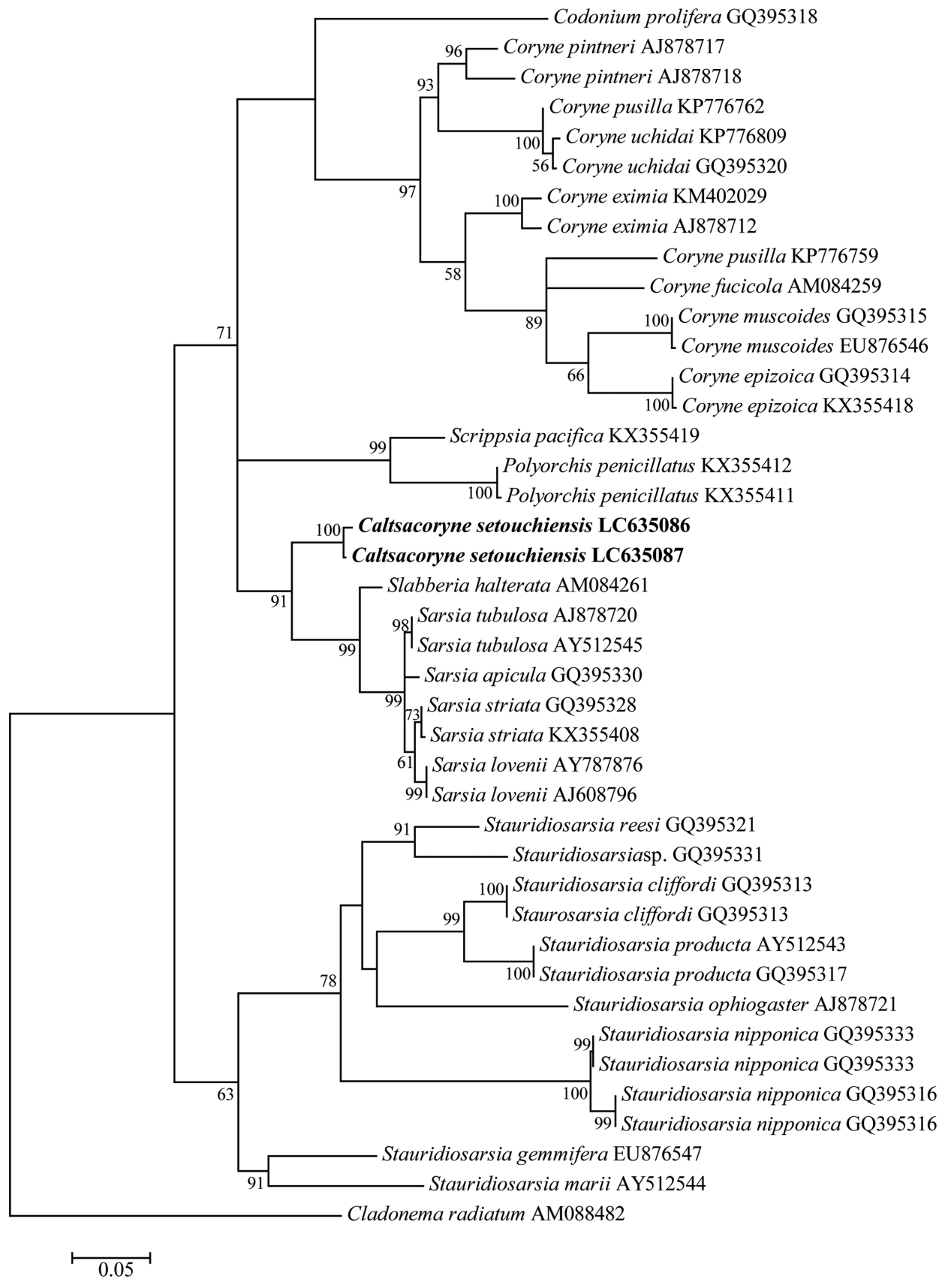

FIGURE 3. Nuclear $16 \mathrm{~S}$ rDNA Maximum-likelihood tree for 25 anthoathcata taxa based on the General Time Reversible model: Scale bar indicates branch length in substitutions per site. Nodal support values are presented as the ML bootstrap value; only values $>50 \%$ are shown. 
TABLE 3. Pair wise genetic distances (K2P) based on 570 positions of $16 \mathrm{~S}$ sequences among Corynidae: The analysis involved 38 sequences.

\begin{tabular}{|c|c|c|c|c|c|c|c|c|c|c|}
\hline No. & 1 & 2 & 3 & 4 & 5 & 6 & 7 & 8 & 9 & 10 \\
\hline \multicolumn{11}{|l|}{ Caltsacoryne setouchiensis n. sp. LC635086 } \\
\hline Caltsacoryne setouchiensis n. sp. LC635087 & 0.01 & & & & & & & & & \\
\hline Codonium prolifera $\mathrm{GQ} 395318$ & 0.12 & 0.12 & & & & & & & & \\
\hline Coryne epizoica GQ395314 & 0.11 & 0.11 & 0.14 & & & & & & & \\
\hline Coryne epizoica KX355418 & 0.11 & 0.11 & 0.14 & 0.00 & & & & & & \\
\hline Coryne eximia AJ878712 & 0.11 & 0.11 & 0.12 & 0.09 & 0.09 & & & & & \\
\hline Coryne eximia KM402029 & 0.12 & 0.11 & 0.12 & 0.09 & 0.09 & 0.02 & & & & \\
\hline Coryne fucicola AM084259 & 0.13 & 0.13 & 0.14 & 0.08 & 0.08 & 0.08 & 0.08 & & & \\
\hline Coryne muscoides EU876546 & 0.13 & 0.13 & 0.13 & 0.07 & 0.07 & 0.10 & 0.10 & 0.07 & & \\
\hline Coryne muscoides GQ395315 & 0.13 & 0.13 & 0.13 & 0.07 & 0.07 & 0.10 & 0.10 & 0.07 & 0.00 & \\
\hline Coryne pintneri AJ878717 & 0.10 & 0.10 & 0.12 & 0.09 & 0.09 & 0.08 & 0.08 & 0.08 & 0.09 & 0.09 \\
\hline Coryne pintneri AJ878718 & 0.12 & 0.11 & 0.13 & 0.09 & 0.09 & 0.07 & 0.07 & 0.09 & 0.10 & 0.10 \\
\hline Coryne pusilla KP776759 & 0.13 & 0.13 & 0.13 & 0.08 & 0.08 & 0.09 & 0.09 & 0.08 & 0.08 & 0.08 \\
\hline Coryne pusilla KP776762 & 0.13 & 0.13 & 0.15 & 0.11 & 0.11 & 0.09 & 0.09 & 0.11 & 0.10 & 0.10 \\
\hline Coryne uchidai GQ395320 & 0.13 & 0.12 & 0.14 & 0.11 & 0.11 & 0.09 & 0.09 & 0.10 & 0.10 & 0.10 \\
\hline Coryne uchidai KP776809 & 0.13 & 0.13 & 0.14 & 0.11 & 0.11 & 0.09 & 0.09 & 0.11 & 0.11 & 0.11 \\
\hline Polyorchis penicillatus KX355411 & 0.09 & 0.09 & 0.15 & 0.15 & 0.15 & 0.15 & 0.15 & 0.16 & 0.16 & 0.16 \\
\hline Polyorchis penicillatus KX355412 & 0.09 & 0.09 & 0.15 & 0.14 & 0.14 & 0.15 & 0.15 & 0.16 & 0.16 & 0.16 \\
\hline Sarsia apicula GQ395330 & 0.07 & 0.07 & 0.12 & 0.14 & 0.14 & 0.13 & 0.14 & 0.15 & 0.15 & 0.15 \\
\hline Sarsia lovenii AJ608796 & 0.06 & 0.06 & 0.12 & 0.13 & 0.13 & 0.13 & 0.13 & 0.15 & 0.15 & 0.15 \\
\hline Sarsia lovenii AY787876 & 0.06 & 0.06 & 0.12 & 0.13 & 0.13 & 0.13 & 0.13 & 0.15 & 0.15 & 0.15 \\
\hline Sarsia striata GQ395328 & 0.06 & 0.06 & 0.12 & 0.13 & 0.13 & 0.12 & 0.13 & 0.14 & 0.14 & 0.14 \\
\hline Sarsia striata KX355408 & 0.06 & 0.06 & 0.11 & 0.13 & 0.13 & 0.12 & 0.13 & 0.14 & 0.15 & 0.15 \\
\hline Sarsia tubulosa AJ878720 & 0.06 & 0.07 & 0.11 & 0.14 & 0.14 & 0.13 & 0.13 & 0.15 & 0.15 & 0.15 \\
\hline Sarsia tubulosa AY512545 & 0.06 & 0.07 & 0.11 & 0.14 & 0.14 & 0.13 & 0.13 & 0.15 & 0.15 & 0.15 \\
\hline Scrippsia pacifica KX355419 & 0.10 & 0.10 & 0.15 & 0.14 & 0.14 & 0.13 & 0.12 & 0.15 & 0.16 & 0.16 \\
\hline Slabberia halterata AM084261 & 0.06 & 0.06 & 0.10 & 0.12 & 0.12 & 0.11 & 0.11 & 0.13 & 0.13 & 0.13 \\
\hline Stauridiosarsia cliffordi GQ395313 & 0.14 & 0.14 & 0.17 & 0.18 & 0.18 & 0.14 & 0.15 & 0.18 & 0.19 & 0.19 \\
\hline Stauridiosarsia gemmifera EU876547 & 0.12 & 0.12 & 0.17 & 0.14 & 0.14 & 0.14 & 0.15 & 0.16 & 0.16 & 0.16 \\
\hline Stauridiosarsia marii AY512544 & 0.13 & 0.13 & 0.17 & 0.15 & 0.15 & 0.14 & 0.14 & 0.16 & 0.16 & 0.16 \\
\hline Stauridiosarsia nipponica GQ395316 & 0.17 & 0.17 & 0.17 & 0.18 & 0.17 & 0.17 & 0.17 & 0.17 & 0.18 & 0.18 \\
\hline Stauridiosarsia nipponica GQ395333 & 0.17 & 0.17 & 0.17 & 0.18 & 0.18 & 0.17 & 0.17 & 0.17 & 0.19 & 0.19 \\
\hline Stauridiosarsia ophiogaster AJ878721 & 0.18 & 0.17 & 0.17 & 0.19 & 0.19 & 0.16 & 0.17 & 0.21 & 0.20 & 0.20 \\
\hline Stauridiosarsia producta AY512543 & 0.12 & 0.12 & 0.17 & 0.17 & 0.17 & 0.15 & 0.15 & 0.18 & 0.20 & 0.20 \\
\hline Stauridiosarsia producta GQ395317 & 0.12 & 0.12 & 0.17 & 0.17 & 0.17 & 0.15 & 0.15 & 0.18 & 0.20 & 0.20 \\
\hline Stauridiosarsia reesi GQ395321 & 0.13 & 0.13 & 0.16 & 0.16 & 0.16 & 0.15 & 0.15 & 0.17 & 0.17 & 0.17 \\
\hline Stauridiosarsia sp. GQ395331 & 0.12 & 0.12 & 0.16 & 0.15 & 0.15 & 0.15 & 0.15 & 0.17 & 0.18 & 0.18 \\
\hline Cladonema radiatum AM088482 & 0.17 & 0.16 & 0.17 & 0.17 & 0.17 & 0.15 & 0.15 & 0.15 & 0.17 & 0.17 \\
\hline
\end{tabular}


TABLE 3. (continued)

\begin{tabular}{|c|c|c|c|c|c|c|c|c|c|c|}
\hline No. & 11 & 12 & 13 & 14 & 15 & 16 & 17 & 18 & 19 & 20 \\
\hline \multicolumn{11}{|l|}{$\begin{array}{l}\text { Caltsacoryne setouchiensis n. sp. } \\
\text { LC635086 }\end{array}$} \\
\hline \multicolumn{11}{|l|}{$\begin{array}{l}\text { Caltsacoryne setouchiensis n. sp. } \\
\text { LC635087 }\end{array}$} \\
\hline \multicolumn{11}{|l|}{ Codonium prolifera GQ395318 } \\
\hline \multicolumn{11}{|l|}{ Coryne epizoica GQ395314 } \\
\hline \multicolumn{11}{|l|}{ Coryne epizoica KX355418 } \\
\hline \multicolumn{11}{|l|}{ Coryne eximia AJ878712 } \\
\hline \multicolumn{11}{|l|}{ Coryne eximia KM402029 } \\
\hline \multicolumn{11}{|l|}{ Coryne fucicola AM084259 } \\
\hline \multicolumn{11}{|l|}{ Coryne muscoides EU876546 } \\
\hline \multicolumn{11}{|l|}{ Coryne muscoides GQ395315 } \\
\hline \multicolumn{11}{|l|}{ Coryne pintneri AJ878717 } \\
\hline Coryne pintneri AJ878718 & 0.03 & & & & & & & & & \\
\hline Coryne pusilla KP776759 & 0.09 & 0.09 & & & & & & & & \\
\hline Coryne pusilla KP776762 & 0.06 & 0.07 & 0.11 & & & & & & & \\
\hline Coryne uchidai GQ395320 & 0.06 & 0.07 & 0.11 & 0.01 & & & & & & \\
\hline Coryne uchidai KP776809 & 0.06 & 0.07 & 0.11 & 0.01 & 0.01 & & & & & \\
\hline Polyorchis penicillatus KX355411 & 0.12 & 0.15 & 0.17 & 0.15 & 0.14 & 0.15 & & & & \\
\hline Polyorchis penicillatus KX355412 & 0.12 & 0.14 & 0.16 & 0.15 & 0.14 & 0.15 & 0.00 & & & \\
\hline Sarsia apicula GQ395330 & 0.12 & 0.13 & 0.15 & 0.13 & 0.12 & 0.12 & 0.12 & 0.12 & & \\
\hline Sarsia lovenii AJ608796 & 0.12 & 0.12 & 0.15 & 0.13 & 0.13 & 0.13 & 0.12 & 0.12 & 0.02 & \\
\hline Sarsia lovenii AY787876 & 0.12 & 0.12 & 0.15 & 0.13 & 0.13 & 0.13 & 0.12 & 0.12 & 0.02 & 0.00 \\
\hline Sarsia striata GQ395328 & 0.12 & 0.12 & 0.14 & 0.12 & 0.12 & 0.12 & 0.11 & 0.11 & 0.01 & 0.01 \\
\hline Sarsia striata KX355408 & 0.12 & 0.12 & 0.14 & 0.12 & 0.12 & 0.12 & 0.11 & 0.11 & 0.01 & 0.01 \\
\hline Sarsia tubulosa AJ878720 & 0.12 & 0.12 & 0.15 & 0.13 & 0.12 & 0.12 & 0.12 & 0.12 & 0.01 & 0.01 \\
\hline Sarsia tubulosa AY512545 & 0.12 & 0.12 & 0.15 & 0.13 & 0.12 & 0.12 & 0.12 & 0.12 & 0.01 & 0.01 \\
\hline Scrippsia pacifica KX355419 & 0.13 & 0.14 & 0.16 & 0.14 & 0.14 & 0.14 & 0.07 & 0.07 & 0.12 & 0.13 \\
\hline Slabberia halterata AM084261 & 0.10 & 0.11 & 0.13 & 0.12 & 0.11 & 0.11 & 0.11 & 0.11 & 0.03 & 0.04 \\
\hline Stauridiosarsia cliffordi GQ395313 & 0.15 & 0.16 & 0.18 & 0.18 & 0.18 & 0.18 & 0.16 & 0.15 & 0.15 & 0.15 \\
\hline Stauridiosarsia gemmifera EU876547 & 0.11 & 0.12 & 0.15 & 0.14 & 0.14 & 0.14 & 0.14 & 0.14 & 0.14 & 0.14 \\
\hline Stauridiosarsia marii AY512544 & 0.12 & 0.13 & 0.16 & 0.13 & 0.13 & 0.14 & 0.16 & 0.15 & 0.14 & 0.14 \\
\hline Stauridiosarsia nipponica GQ395316 & 0.15 & 0.15 & 0.17 & 0.17 & 0.17 & 0.17 & 0.18 & 0.17 & 0.17 & 0.17 \\
\hline Stauridiosarsia nipponica GQ395333 & 0.15 & 0.15 & 0.17 & 0.17 & 0.17 & 0.17 & 0.17 & 0.17 & 0.16 & 0.16 \\
\hline Stauridiosarsia ophiogaster AJ878721 & 0.15 & 0.16 & 0.17 & 0.18 & 0.18 & 0.19 & 0.19 & 0.19 & 0.18 & 0.19 \\
\hline Stauridiosarsia producta AY512543 & 0.15 & 0.16 & 0.18 & 0.18 & 0.18 & 0.19 & 0.15 & 0.15 & 0.15 & 0.14 \\
\hline Stauridiosarsia producta GQ395317 & 0.15 & 0.16 & 0.18 & 0.18 & 0.18 & 0.19 & 0.15 & 0.15 & 0.15 & 0.14 \\
\hline Stauridiosarsia reesi GQ395321 & 0.15 & 0.16 & 0.16 & 0.16 & 0.16 & 0.16 & 0.15 & 0.15 & 0.13 & 0.13 \\
\hline Stauridiosarsia sp. GQ395331 & 0.14 & 0.15 & 0.17 & 0.16 & 0.16 & 0.16 & 0.13 & 0.13 & 0.13 & 0.13 \\
\hline Cladonema radiatum AM088482 & 0.15 & 0.16 & 0.17 & 0.17 & 0.17 & 0.17 & 0.17 & 0.17 & 0.18 & 0.18 \\
\hline
\end{tabular}


TABLE 3. (continued)

\begin{tabular}{|c|c|c|c|c|c|c|c|c|c|c|}
\hline No. & 21 & 22 & 23 & 24 & 25 & 26 & 27 & 28 & 29 & 30 \\
\hline \multicolumn{11}{|l|}{$\begin{array}{l}\text { Caltsacoryne setouchiensis } \mathrm{n} . \mathrm{sp} \text {. } \\
\text { LC635086 }\end{array}$} \\
\hline \multicolumn{11}{|l|}{$\begin{array}{l}\text { Caltsacoryne setouchiensis n. sp. } \\
\text { LC635087 }\end{array}$} \\
\hline \multicolumn{11}{|l|}{ Codonium prolifera GQ395318 } \\
\hline \multicolumn{11}{|l|}{ Coryne epizoica GQ395314 } \\
\hline \multicolumn{11}{|l|}{ Coryne epizoica KX355418 } \\
\hline \multicolumn{11}{|l|}{ Coryne eximia AJ878712 } \\
\hline \multicolumn{11}{|l|}{ Coryne eximia KM402029 } \\
\hline \multicolumn{11}{|l|}{ Coryne fucicola AM084259 } \\
\hline \multicolumn{11}{|l|}{ Coryne muscoides EU876546 } \\
\hline \multicolumn{11}{|l|}{ Coryne muscoides GQ395315 } \\
\hline \multicolumn{11}{|l|}{ Coryne pintneri AJ878717 } \\
\hline \multicolumn{11}{|l|}{ Coryne pintneri AJ878718 } \\
\hline \multicolumn{11}{|l|}{ Coryne pusilla KP776759 } \\
\hline \multicolumn{11}{|l|}{ Coryne pusilla KP776762 } \\
\hline \multicolumn{11}{|l|}{ Coryne uchidai GQ395320 } \\
\hline \multicolumn{11}{|l|}{ Coryne uchidai KP776809 } \\
\hline \multicolumn{11}{|l|}{ Polyorchis penicillatus KX355411 } \\
\hline \multicolumn{11}{|l|}{ Polyorchis penicillatus KX355412 } \\
\hline \multicolumn{11}{|l|}{ Sarsia apicula GQ395330 } \\
\hline \multicolumn{11}{|l|}{ Sarsia lovenii AJ608796 } \\
\hline \multicolumn{11}{|l|}{ Sarsia lovenii AY787876 } \\
\hline Sarsia striata GQ395328 & 0.01 & & & & & & & & & \\
\hline Sarsia striata KX355408 & 0.01 & 0.00 & & & & & & & & \\
\hline Sarsia tubulosa AJ878720 & 0.01 & 0.01 & 0.01 & & & & & & & \\
\hline Sarsia tubulosa AY512545 & 0.01 & 0.01 & 0.01 & 0.00 & & & & & & \\
\hline Scrippsia pacifica KX355419 & 0.13 & 0.12 & 0.11 & 0.12 & 0.12 & & & & & \\
\hline Slabberia halterata AM084261 & 0.04 & 0.04 & 0.04 & 0.03 & 0.03 & 0.11 & & & & \\
\hline Stauridiosarsia cliffordi GQ395313 & 0.15 & 0.14 & 0.14 & 0.14 & 0.14 & 0.15 & 0.13 & & & \\
\hline Stauridiosarsia gemmifera EU876547 & 0.14 & 0.14 & 0.14 & 0.14 & 0.14 & 0.13 & 0.12 & 0.12 & & \\
\hline Stauridiosarsia marii AY512544 & 0.14 & 0.14 & 0.13 & 0.14 & 0.14 & 0.14 & 0.11 & 0.12 & 0.09 & \\
\hline Stauridiosarsia nipponica GQ395316 & 0.17 & 0.17 & 0.17 & 0.17 & 0.17 & 0.18 & 0.17 & 0.14 & 0.14 & 0.15 \\
\hline Stauridiosarsia nipponica GQ395333 & 0.16 & 0.16 & 0.16 & 0.16 & 0.16 & 0.18 & 0.17 & 0.14 & 0.14 & 0.15 \\
\hline Stauridiosarsia ophiogaster AJ878721 & 0.19 & 0.19 & 0.19 & 0.18 & 0.18 & 0.19 & 0.17 & 0.12 & 0.12 & 0.16 \\
\hline Stauridiosarsia producta AY512543 & 0.14 & 0.14 & 0.14 & 0.14 & 0.14 & 0.15 & 0.14 & 0.05 & 0.13 & 0.12 \\
\hline Stauridiosarsia producta GQ395317 & 0.14 & 0.14 & 0.14 & 0.14 & 0.14 & 0.15 & 0.14 & 0.05 & 0.13 & 0.12 \\
\hline Stauridiosarsia reesi GQ395321 & 0.13 & 0.13 & 0.13 & 0.13 & 0.13 & 0.16 & 0.13 & 0.08 & 0.13 & 0.12 \\
\hline Stauridiosarsia sp. GQ395331 & 0.13 & 0.12 & 0.12 & 0.12 & 0.12 & 0.15 & 0.12 & 0.10 & 0.12 & 0.12 \\
\hline Cladonema radiatum AM088482 & 0.18 & 0.17 & 0.17 & 0.18 & 0.18 & 0.16 & 0.16 & 0.15 & 0.19 & 0.16 \\
\hline
\end{tabular}


TABLE 3. (continued)

\begin{tabular}{|c|c|c|c|c|c|c|c|c|}
\hline No. & 31 & 32 & 33 & 34 & 35 & 36 & 37 & 38 \\
\hline \multicolumn{9}{|c|}{ Caltsacoryne setouchiensis n. sp. LC635086 } \\
\hline \multicolumn{9}{|c|}{ Caltsacoryne setouchiensis n. sp. LC635087 } \\
\hline \multicolumn{9}{|c|}{ Codonium prolifera GQ395318 } \\
\hline \multicolumn{9}{|l|}{ Coryne epizoica GQ395314 } \\
\hline \multicolumn{9}{|l|}{ Coryne epizoica KX355418 } \\
\hline \multicolumn{9}{|l|}{ Coryne eximia AJ878712 } \\
\hline \multicolumn{9}{|l|}{ Coryne eximia KM402029 } \\
\hline \multicolumn{9}{|l|}{ Coryne fucicola AM084259 } \\
\hline \multicolumn{9}{|l|}{ Coryne muscoides EU876546 } \\
\hline \multicolumn{9}{|l|}{ Coryne muscoides GQ395315 } \\
\hline \multicolumn{9}{|l|}{ Coryne pintneri AJ878717 } \\
\hline \multicolumn{9}{|l|}{ Coryne pintneri AJ878718 } \\
\hline \multicolumn{9}{|l|}{ Coryne pusilla KP776759 } \\
\hline \multicolumn{9}{|l|}{ Coryne pusilla KP776762 } \\
\hline \multicolumn{9}{|l|}{ Coryne uchidai GQ395320 } \\
\hline \multicolumn{9}{|l|}{ Coryne uchidai KP776809 } \\
\hline \multicolumn{9}{|l|}{ Polyorchis penicillatus KX355411 } \\
\hline \multicolumn{9}{|l|}{ Polyorchis penicillatus KX355412 } \\
\hline \multicolumn{9}{|l|}{ Sarsia apicula GQ395330 } \\
\hline \multicolumn{9}{|l|}{ Sarsia lovenii AJ608796 } \\
\hline \multicolumn{9}{|l|}{ Sarsia lovenii AY787876 } \\
\hline \multicolumn{9}{|l|}{ Sarsia striata GQ395328 } \\
\hline \multicolumn{9}{|l|}{ Sarsia striata KX355408 } \\
\hline \multicolumn{9}{|l|}{ Sarsia tubulosa AJ878720 } \\
\hline \multicolumn{9}{|l|}{ Sarsia tubulosa AY512545 } \\
\hline Scrippsia pacifica KX355419 & & & & & & & & \\
\hline Slabberia halterata AM084261 & & & & & & & & \\
\hline Stauridiosarsia cliffordi GQ395313 & & & & & & & & \\
\hline Stauridiosarsia gemmifera EU876547 & & & & & & & & \\
\hline Stauridiosarsia marii AY512544 & & & & & & & & \\
\hline Stauridiosarsia nipponica GQ395316 & & & & & & & & \\
\hline Stauridiosarsia nipponica GQ395333 & 0.01 & & & & & & & \\
\hline Stauridiosarsia ophiogaster AJ878721 & 0.13 & 0.13 & & & & & & \\
\hline Stauridiosarsia producta AY512543 & 0.14 & 0.14 & 0.11 & & & & & \\
\hline Stauridiosarsia producta GQ395317 & 0.14 & 0.14 & 0.11 & 0.00 & & & & \\
\hline Stauridiosarsia reesi GQ395321 & 0.12 & 0.12 & 0.12 & 0.09 & 0.09 & & & \\
\hline Stauridiosarsia sp. GQ395331 & 0.12 & 0.11 & 0.12 & 0.10 & 0.10 & 0.06 & & \\
\hline Cladonema radiatum AM088482 & 0.16 & 0.16 & 0.19 & 0.16 & 0.16 & 0.16 & 0.16 & \\
\hline
\end{tabular}


Taxonomy

Phylum Cnidaria Verrill, 1865

Subphylum Medusozoa Petersen, 1979

Class Hydrozoa Owen, 1843

Subclass Hydroidolina Collins, 2000

Order Anthoathecata Cornelius, 1992

\section{Suborder Capitata Kühn, 1913}

\section{Family Corynidae Johnston, 1836}

\section{Genus Caltsacoryne gen. n.}

New Japanese name: Shitouzu-kurage-zoku

Genus diagnosis. Corynidae have a bell-shaped umbrella with four radial canals, and a ring canal. Tentacle bulbs swollen, each with an abaxial ocellus. Manubrium short, not extending beyond umbrella margin and without thin proximal part. Gonads undivided, and encircle manubrium for nearly its entire length, leaving manubrium visible only at the top and near the mouth. Four tentacles arising from bulbs with a single egg-shaped nematocyst swelling at tips.

Type species. Caltsacoryne setouchiensis

Etymology. The genus name Caltsacoryne is taken from the Greek words "kaltsa" and "koryne," meaning "sock" and "club," respectively. The gender is feminine. The name reflects the illusion that the medusa appears to be wearing socks on the tentacle tips.

\section{Caltsacoryne setouchiensis sp. $\mathrm{n}$.}

New Japanese name: Shitouzu-kurage

Figures 4-7

Material examined. Holotype: NSMT-Co 1749. Suo-Oshima, Okikamuro Island, Yamaguchi Prefecture, western Japan, April 24, 2016, collector: Sho Toshino, one adult female medusa. Paratypes. NSMT-Co 1750. Same locality and date as the holotype, collectors: Yoshimi Hamatsu, one adult male. NSMT-Co1751-1753. Same locality as the holotype, April 18, 2018, collector: Hiroaki Uchida, two adult males and one adult female.

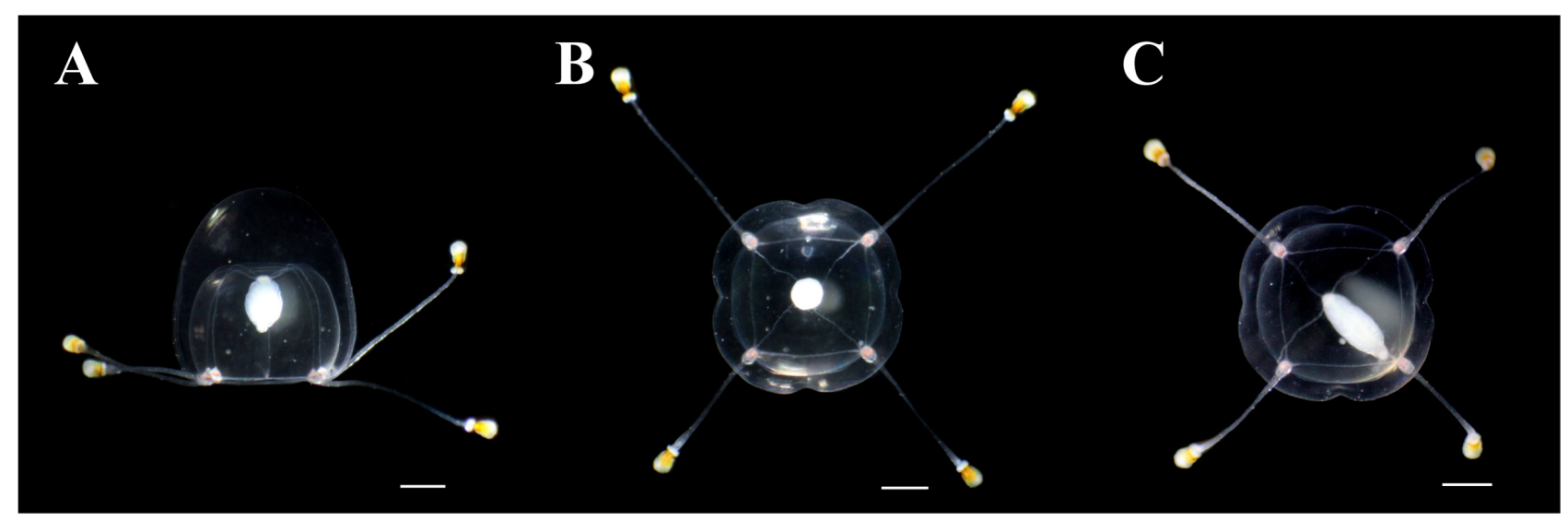

FIGURE 4. Caltsacoryne setouchiensis sp. n., live: A, lateral view; B, apical view; C, oral view. All bars represent 1 mm. 
Description. Mature medusae with transparent, thick but soft bell-shaped umbrella (Fig. 4A-C, 5A-C). Umbrella height (UH) and diameter (UD) approximately $7 \mathrm{~mm}$ and $4 \mathrm{~mm}$, respectively (Table 4). Umbrella apex thickened, tapered. Exumbrella smooth, nematocyst sparsely scattered. Bell cavity small. Four radial canals and single straight ring canal. Canals narrow, almost with same diameter. Tentacle bulbs swollen, reddish purple in color, each with abaxial ocellus (Fig. 6A-C). Tentacle arising from bulbs, short and terminating in single large egg-shaped swelling covered with nematocysts (Fig. 6D, E). Orange band present on middle part of swelling. Mouth simple and circular, whitish in color (Fig. 6F, G). Manubrium hanging in bell cavity, large and flask-shaped; rounded at apical side and furnished with small proboscis in distal portion. Extended manubrium length approximately $3 \mathrm{~mm}$ and approximately half umbrella height (almost full length of subumbrella). Gonads encircling entire surface of manubrium, with exception of apical and distal portions (Fig. 6D). Velum narrow (Fig. 4C, 5C).

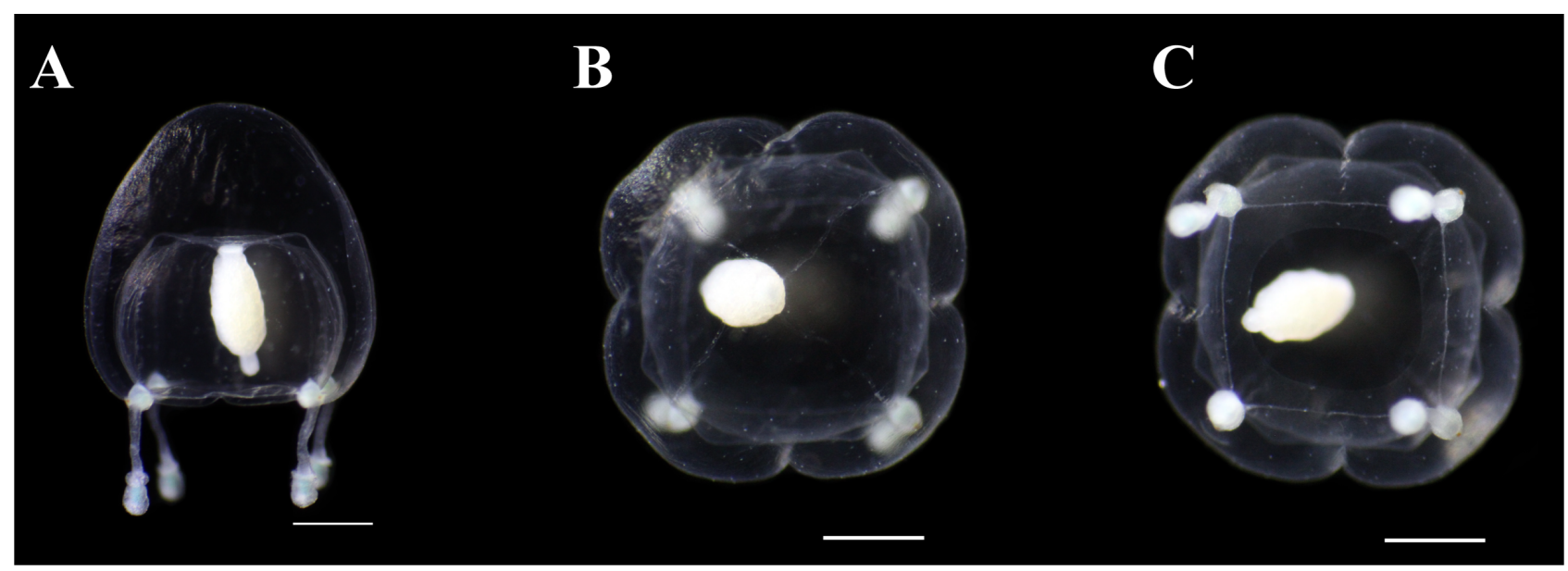

FIGURE 5. Caltsacoryne setouchiensis sp. n., fixed, holotype: A, lateral view; B, apical view; C, oral view. All bars represent 1 mm.

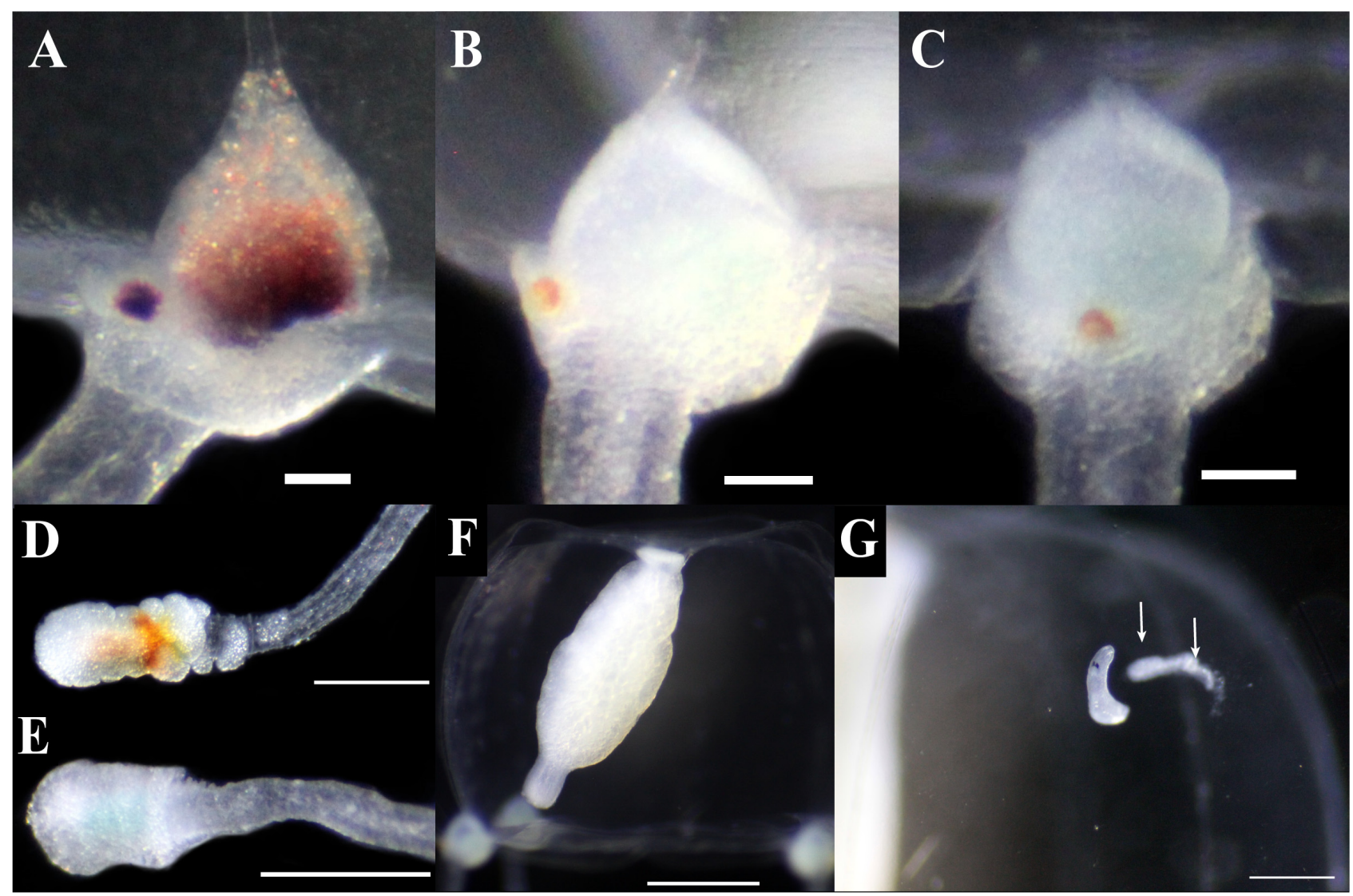

FIGURE 6. Caltsacoryne setouchiensis, sp. n.: A-C, tentacle bulb. Lateral view, live (A), lateral view, fixed (B); abaxial view, fixed (C); D-E, tentacle. Live (D), fixed (E); F, manubrium and gonad; G, exumbrella. Arrows indicate flukes;. Scale bars: 0.1 $\mathrm{mm}(\mathrm{A}-\mathrm{C}), 0.5 \mathrm{~mm}(\mathrm{D}-\mathrm{G})$. 
TABLE 4. Size (mm) of Caltsacoryne setouchiensis: * The holotype. Nos. NSMT-Co1750-1753 are paratypes. ML= manubrium length; $\mathrm{UH}=$ umbrella height; $\mathrm{UD}=$ umbrella width.

\begin{tabular}{llllll}
\hline Specimen No. & UH & UD & ML & Sex & Date \\
\hline NSMT-Co 1749* & 3.7 & 2.8 & 1.8 & Female & $2016 / 4 / 24$ \\
NSMT-Co 1750 & 3.7 & 2.5 & 2.3 & Male & $2016 / 4 / 24$ \\
NSMT-Co 1751 & 6.7 & 3.2 & 2.5 & Male & $2018 / 4 / 18$ \\
NSMT-Co 1752 & 7.3 & 4.0 & 3.6 & Male & $2018 / 4 / 18$ \\
NSMT-Co 1753 & 6.5 & 3.9 & 2.6 & Female & $2018 / 4 / 18$ \\
\hline
\end{tabular}

Smallest young medusa with UH of $2.5 \mathrm{~mm}$ and UD of $2.6 \mathrm{~mm}$ (Fig. 7A-C). Umbrella spherical with few exumbrellar nematocysts. Mesoglea on apex of exumbrella thinner than that of adults. Manubrium thin, translucent to whitish, length approximately half that of umbrella. Mouth simple and circular. Gonad immature. Four radial canals and singular circular canal. Velum narrow. Tentacle bulbs swollen, reddish purple, each with dark brown abaxial ocellus. Four tentacles, terminating in single large spherical swelling. Orange band present on middle part of swelling.

TABLE 5. Cnidomes of Caltsacoryne setouchiensis: D, L represent capsule diameter and length, respectively, in $\mu \mathrm{m}$.

\begin{tabular}{|c|c|c|c|c|c|c|c|}
\hline Part & Type & & Min & Max & Mean & SD & $\mathrm{N}$ \\
\hline \multirow[t]{6}{*}{ Tentacular bulb } & Desmonema & $\mathrm{D}$ & 5.7 & 8.7 & 7.8 & 0.6 & 50 \\
\hline & & $\mathrm{L}$ & 14.6 & 20.3 & 16.9 & 1.1 & \\
\hline & Stenotele (Large) & $\mathrm{D}$ & 14.0 & 22.1 & 17.5 & 2.7 & 31 \\
\hline & & $\mathrm{L}$ & 21.0 & 29.0 & 24.1 & 2.2 & \\
\hline & Stenotele (Small) & $\mathrm{D}$ & 9.0 & 12.9 & 11.0 & 0.7 & 45 \\
\hline & & $\mathrm{L}$ & 15.1 & 19.1 & 17.1 & 0.9 & \\
\hline \multirow[t]{6}{*}{ Tentacle tip } & Desmonema & $\mathrm{D}$ & 5.0 & 7.2 & 5.9 & 0.7 & 17 \\
\hline & & $\mathrm{L}$ & 8.9 & 15.3 & 11.5 & 1.7 & \\
\hline & Stenotele (Large) & $\mathrm{D}$ & 16.0 & 20.3 & 18.1 & 1.0 & 50 \\
\hline & & $\mathrm{L}$ & 21.5 & 26.2 & 23.7 & 1.0 & \\
\hline & Stenotele (Small) & $\mathrm{D}$ & 9.9 & 12.0 & 11.2 & 0.6 & 31 \\
\hline & & $\mathrm{L}$ & 14.6 & 17.9 & 16.2 & 0.8 & \\
\hline \multirow[t]{4}{*}{ Manubrium } & Desmonema & $\mathrm{D}$ & 5.1 & 9.4 & 7.2 & 0.8 & 49 \\
\hline & & $\mathrm{L}$ & 9.6 & 17.2 & 14.4 & 1.3 & \\
\hline & Stenotele (Large) & $\mathrm{D}$ & 15.2 & 20.8 & 18.0 & 1.9 & 17 \\
\hline & & $\mathrm{L}$ & 20.8 & 27.8 & 24.8 & 2.1 & \\
\hline
\end{tabular}

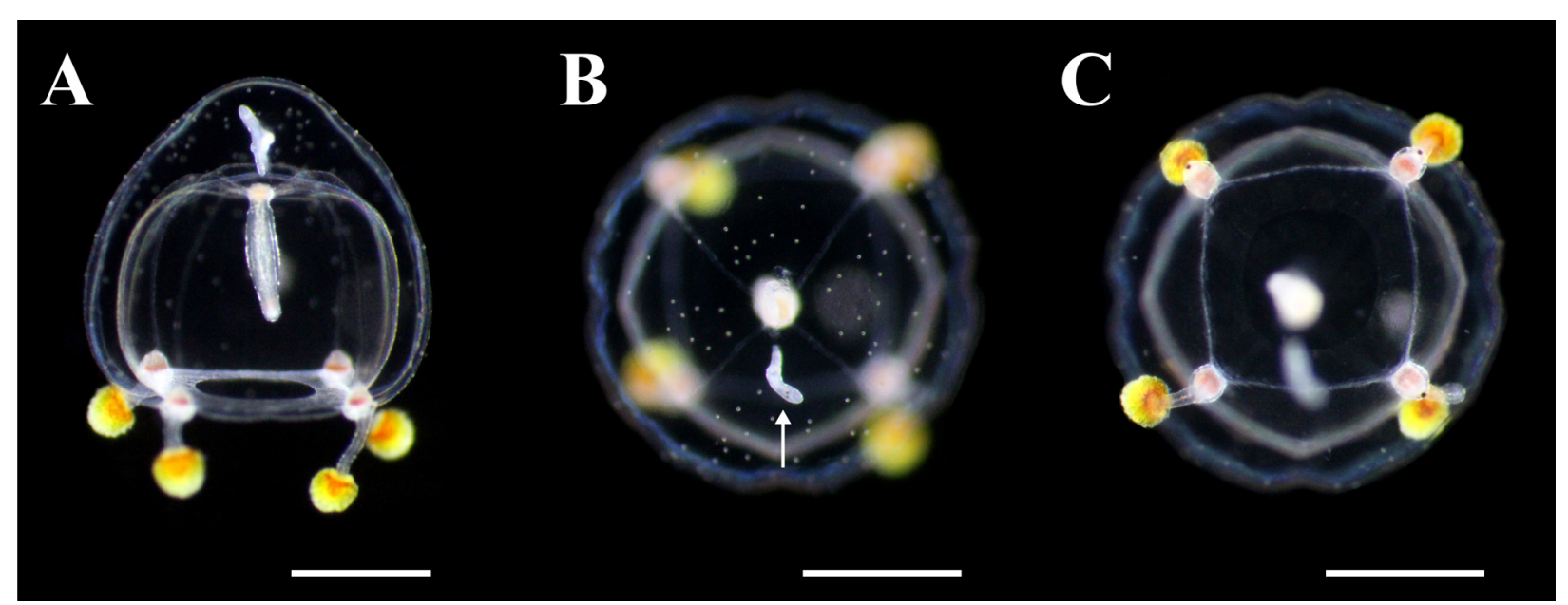

FIGURE 7. Young medusae of Caltsacoryne setouchiensis sp. n., live: A, lateral view; B, apical view; C, oral view. Arrow indicates fluke. All bars represent $1 \mathrm{~mm}$. 


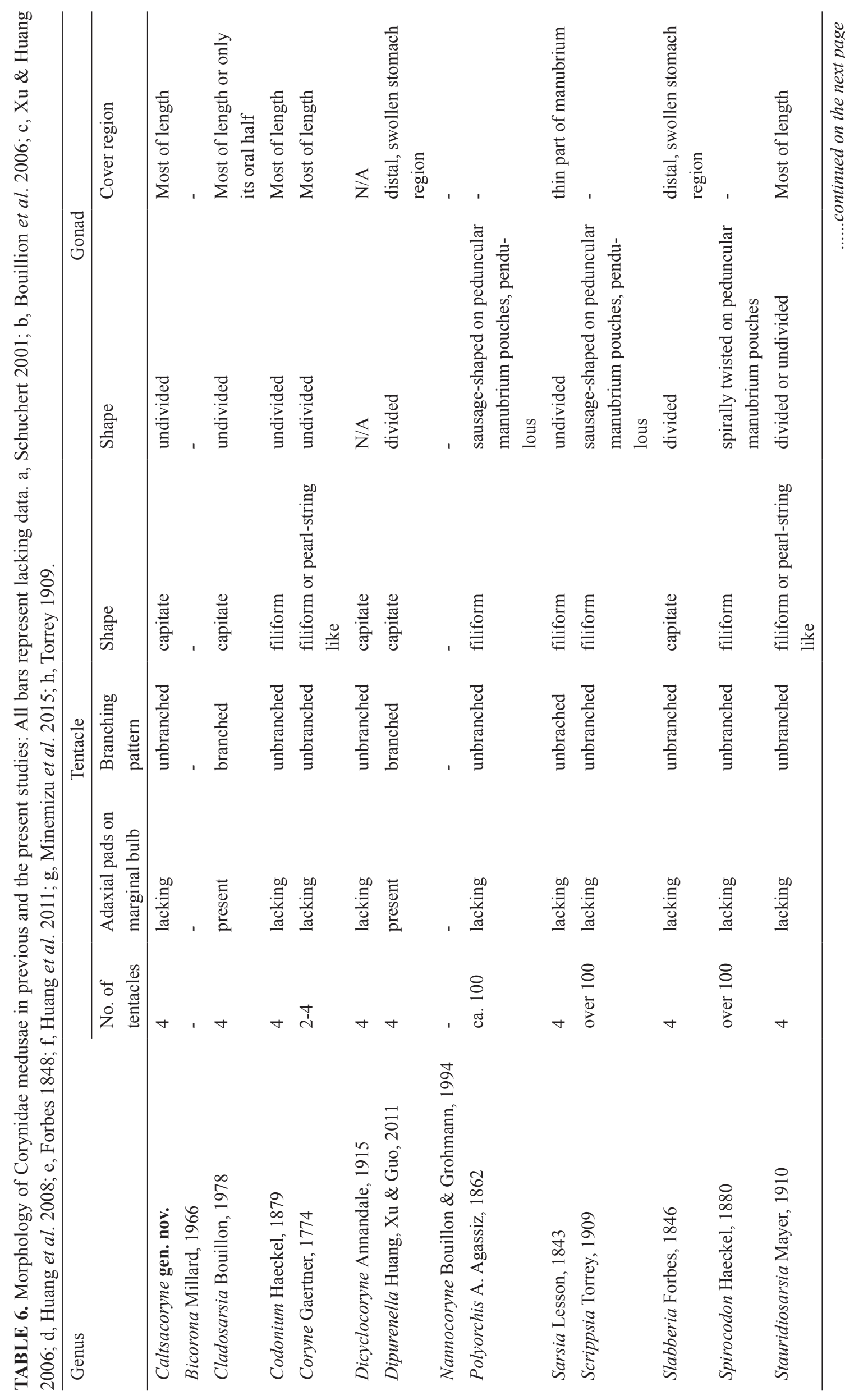




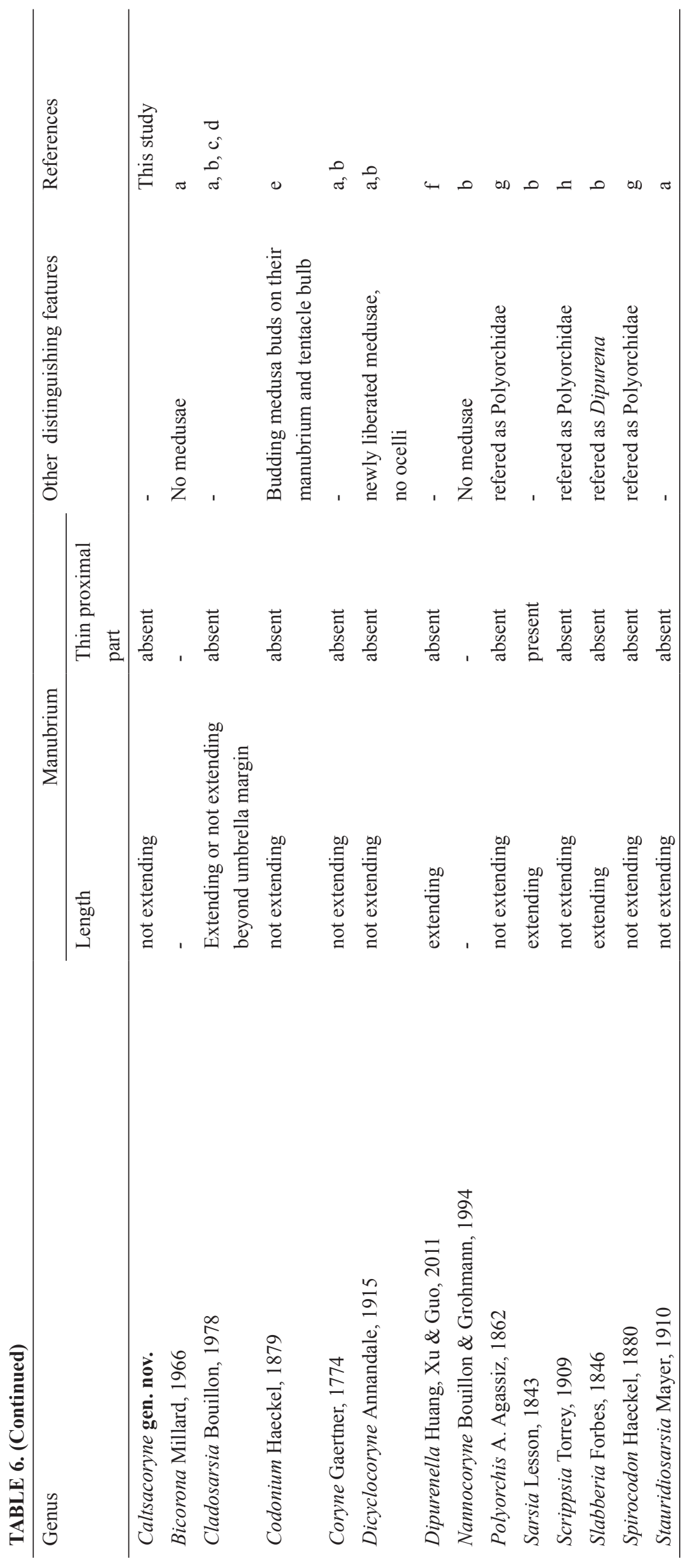


Cnidome. Two different types of nematocyst were identified and measured in the adult medusa (Table 5, Fig. 8), and two sizes of stenoteles (Fig. 8A, B) and desmonemes (Fig. 8C, D) were observed on tentacle tips, tentacle bulbs, and manubrium, although small stenoteles were absent from the manubrium. Stenoteles were also found on the bell; however, these could not be measured.

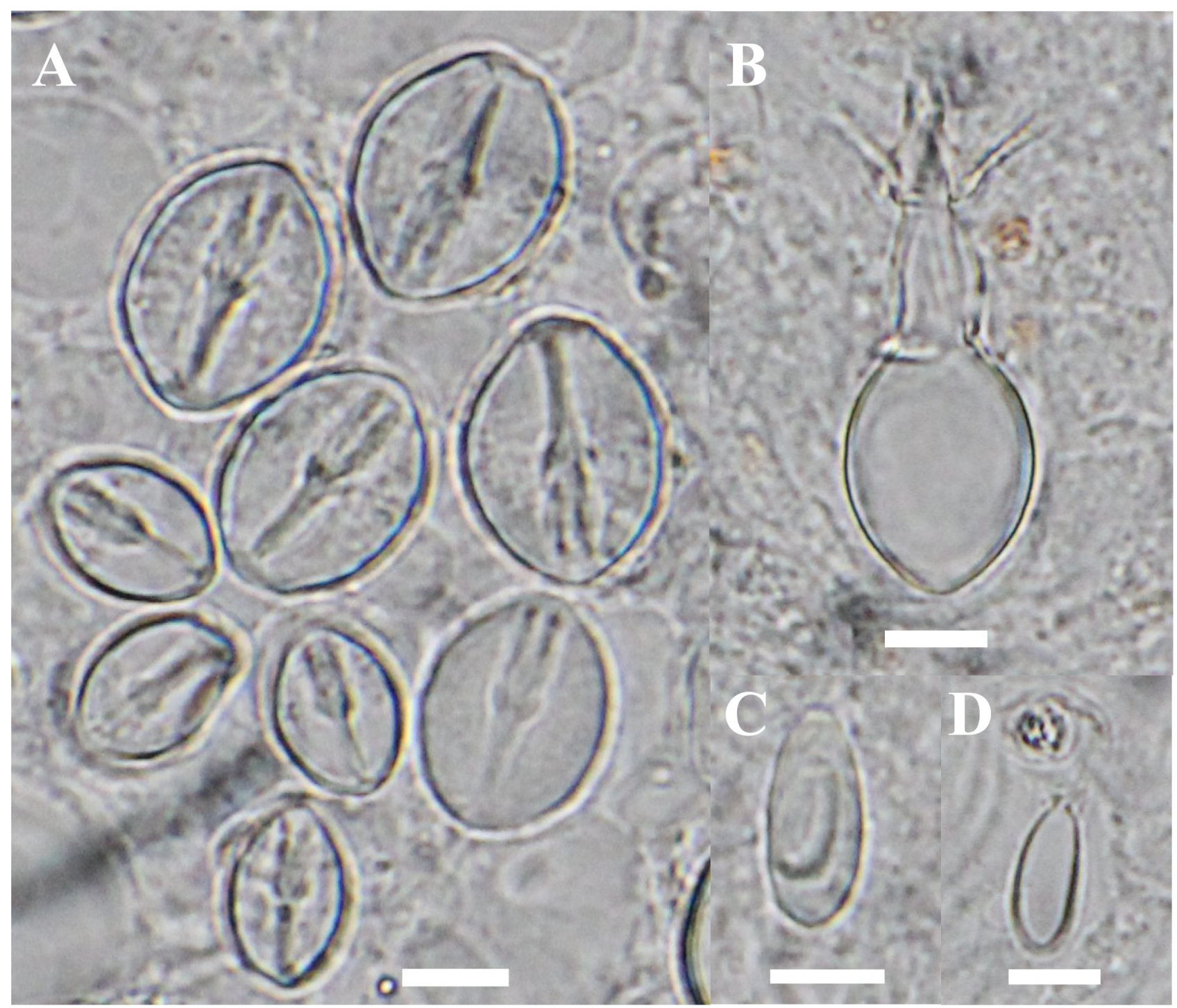

FIGURE 8. Nematocysts of Caltsacoryne setouchiensis, sp. n.: A-B, stenoteles. Intact (A), discharged (B); C-D, desmonemas. Intact (C), discharged (D). All bars represent $10 \mu \mathrm{m}$.

Habitat and ecology. The medusae of $C$. setouchiensis collected in the present study appeared at the water surface in a shallow area (water depth 3-5 m) during the daytime around the coast of Suo-Oshima, the Seto Inland Sea, in April. They are not active swimmers but drift with the current using their extended tentacles. The species appears to be neritic. In some specimens, mesoglea at the apical part were found to be infested by flukes (Fig. 6G, 7B). Stinging events attributable to C. setouchiensis are currently unknown.

Etymology. The specific name "setouchiensis" refers to the Setouchi Region, which includes the type locality in which the species was found.

Differential diagnosis. A comparison of the key features of Corynidae species is presented in Table 6. Caltsacoryne setouchiensis can be distinguished from the species of other genera by the following combination of morphological characters: number of tentacles, cnidocyst pads, manubrium length, and shape of the gonads and tentacles in adult medusae. All species in the family Corynidae have four radial canals, circular canals, and marginal tentacle bulbs. Caltsacoryne bears four tentacles, whereas species in the genera Polyorchis, Scrippsia, and Spirocodon typically have more than 100 . Whereas most corynids have unbranched filiform tentacles, those in Caltsacoryne, Dicyclocoryne, and Slabberia are unbranched capitate, and Cladosarsia and Dipurenella have branched 
capitate tentacles. Caltsacoryne lacks adaxial cnidocyst pads on the marginal bulbs, whereas these pads are present in Cladosarsia and Dipurenella. Furthermore, unlike species of Cladosarsia, Dipurenella, Sarsia and Slabberia, the manubrium in Caltsacoryne does not extend beyond the umbrella margin, and in contrast to Dipurenella, Slabberia, and some species of Stauridiosarsia, in which the gonads are divided, those in Caltsacoryne are divided

\section{Discussion and conclusions}

The morphological characters of C. setouchiensis tend to resemble those of Coryne inabai (Uchida, 1933) and Plotocnida borealis Wagner, 1885. Coryne inabai was originally described by Uchida (1933) as Sarsia inabai collected from the western coast of Kamchatka. However, Kramp (1942; 1968) doubted the validity of this assignment and referred it to Plotocnida borealis Wagner, 1885, despite the lack of ocelli in the marginal tentacle bulbs. Bouillon (1978) reported a mature $1.3 \mathrm{~mm}$ medusa of C. inabai (as Sarsia inabai) from the Seychelles. However, Schuchert (2001) pointed out that the specimen described by Bouillon is probably a different species, given that Uchida (1933) originally described the medusae of $C$. inabai as maturing at $3-8.5 \mathrm{~mm}$; moreover, it also has a slight peduncle. Schuchert (2001) also re-examined a medusa collected by Bouillion (1985b) from Papua New Guinea, and identified the specimen as Coryne inabai. Unfortunately, it is likely that the material investigated for the original description of $C$. inabai has been lost and thus cannot be re-examined.

The specimens of Caltsacoryne setouchiensis were collected from the Seto Inland Sea, western Japan, whereas C. inabai has been reported from the cold northern Pacific (Uchida 1933) and in the tropical Pacific and Indian oceans (Bouillon 1978; 1985; Schuchert 2001), and thus the distribution of these two species would not appear to overlap.

The morphological and molecular phylogenetic analyses undertaken in the present study provide convincing evidence that the Corynidae collected from the Seto Inland Sea is a new genus and new species. Currently, however, the development, seasonal distribution, and stings of this species have yet to be determined. Accordingly, further investigations will be necessary to gain an insight into the ecology of C. setouchiensis.

\section{Acknowledgements}

We would like to express our sincere thanks to Junko Fukada, Takuma Mezaki, Keita Koeda, Tatsuki Koido, Reina Tsujimoto, Takeru Yoshioka, Takaya Kitamura, Chika Nagaoka (Kuroshio Biological Research Foundation), Takashi Asahida, Kenichi Hayashizaki, Hiroshi Miyake (Kitasato University), and the staff of the Sesoko Tropical Biosphere Research Center and Nagisa Aquarium. This manuscript was greatly improved by the constructive comments of Rocktim Ramen Das. This research was financially supported by the Showa Seitoku Memorial Foundation and the JSPS KAKENHI Grant Number JP18K14791.

\section{References}

Bouillon, J. (1978) Hydroméduses de l'archipel des Séchelles et du Mozambique. Revue de Zoologie Africaine, 92 (1), $117-$ 172.

Bouillon, J. (1985) Notes additionelles sur les Hydroméduses de la mer de Bismarck (Hydrozoa-Cnidaria). Indo-Malayan Zoology, 2 (2), 245-266.

Brinckmann-Voss, A. (2000) The hydroid and medusa of Sarsia bella sp. nov. (Hydrozoa, Anthoathecatae, Corynidae), with a correction of the 'life cycle' of Polyorchis penicillatus (Eschscholtz). Scientia Marina, 64 (S1), 189-195. https://doi.org/10.3989/scimar.2000.64s1189

Bouillon, J., Gravili, C., Gili, J.M. \& Boero, F. (2006) An introduction to Hydrozoa. Mémoires du Muséum National d'Histoire Naturelle, 194, 1-591.

Collins, A.G., Winkelmann, S., Hadrys, H. \& Schierwater, B. (2005) Phylogeny of Capitata and Corynidae (Cnidaria, Hydrozoa) in light of mitochondrial 16S rDNA data. Zoologica Scripta, 34 (1), 91-99. https://doi.org/10.1111/j.1463-6409.2005.00172.x

Collins, A.G., Bentlage, B., Lindner, A., Lindsay, D., Haddock, S.H.D., Jarms, G., Norenburg, J.L., Jankowski, T. \& Cartwright, P. (2008) Phylogenetics of Trachylina (Cnidaria: Hydrozoa) with new insights on the evolution of some problematical taxa. Journal of the Marine Biological Association of the United Kingdom, 88, 1673-1685. 
https://doi.org/10.1017/S0025315408001732

Cunningham, C.W. \& Buss, L.W. (1993) Molecular evidence for multiple episodes of paedomorphosis in the family Hydractiniidae. Biochemical Systematics and Ecology, 21, 57-69. https://doi.org/10.1016/0305-1978(93)90009-G

Forbes, E. (1848) A monograph of the British naked-eyed medusae: with figures of all the species. Ray society, London, 104 pp. https://doi.org/10.5962/bhl.title.10032

Huang, J.Q., Xu, Z.Z., Lin, J.Z. \& Qiu, M.F. (2008) Three New Species of Anthomedusae (Hydrozoa, Hydroidomedusae) from the Fujian Sea Water. Journal of Xiamen University, Natural Science, 3, 26.

Huang, J.Q., Xu, Z.Z. \& Guo, D. (2011) Specific characteristic of two new species of Hydromedusae in the south of Taiwan Strait. Journal of Oceanography in Taiwan Strait, 29, 1-4.

Johnston, G. (1836) A catalogue of the zoophytes of Berwickshire. History of the Berwickshire Naturalists' Club, 1, 107-108.

Kimura, M. (1980) A simple method for estimating evolutionary rate of base substitutions through comparative studies of nucleotide sequences. Journal of Molecular Evolution, 16, 111-120. https://doi.org/10.1007/BF01731581

Kramp, P.L. (1942) Medusae, in The Godthaab Expedition, 1928. Meddelelser om Grönland, 81 (1), 1-168.

Kramp, P.L. (1959) The Hydromedusae of the Atlantic Ocean and adjacent waters. Dana-Report, 46, 1-283.

Kramp, P.L. (1968) The Hydromedusae of the Pacific and Indian Oceans, sect. II and III. Dana-Report, 72, 1-200.

Kubota, S. (1991) Taxonomic notes on polyp and medusa of Sarsia nipponica Uchida (Hydrozoa: Corynidae) from the type locality in Japan. Publications of the Seto Marine Biological Laboratory, 35 (1-3), 17-23. https://doi.org/10.5134/176173

Kubota, S. (2014) Fascinating jellyfishes in warm water. Kii Minpou, Wakayama, 167 pp. [in Japanese]

Kubota, S. \& Gravili, C. (2007) A list of hydromedusae (excluding Siphonophora, Milleporidae and Actinulidae) in Japan. Nankiseibutsu, 49 (2), 189-204.

Mayer, A.G. (1910) Medusae of the world. Carnegie Institution of Washington, 109, 1-735. https://doi.org/10.5962/bhl.title.159245

Millard, R.L. (1975) Monograph on the Hydroida of southern Africa. Annals of the South African Museum, 68, 1-513.

Minemizu, R., Kubota, S., Hirano, Y. \& Lindsay, D. (2015) A photographic guide to the jellyfishes of Japan. Heibonsha, Tokyo, $360 \mathrm{pp}$.

Nawrocki, A.M., Schuchert, P. \& Cartwright, P. (2009) Phylogenetics and evolution of Capitata (Cnidaria: Hydrozoa), and the systematics of Corynidae. Zoologica Scripta, 39 (3), 290-304. https://doi.org/10.1111/j.1463-6409.2009.00419.x

Östman, C. (2000) A guideline to nematocyst nomenclature and classification, and some notes on the systematic value of nematocysts. Scientia Marina, 64 (Supplement 1), 31-46. https://doi.org/10.3989/scimar.2000.64s131

Petersen, K.W. (1990) Evolution and taxonomy in capitate hydroids and medusae (Cnidaria: Hydrozoa). Zoological Journal of the Linnean Society, 100, 101-231. https://doi.org/10.1111/j.1096-3642.1990.tb01862.x

Rasband, W.S. (2021) ImageJ. U. S. National Institutes of Health, Bethesda, Maryland. Available from: https://imagej.nih.gov/ ij/ (accessed 17 August 2021)

Schuchert, P. (2001) Survey of the family Corynidae (Cnidaria, Hydrozoa). Revue Suisse de Zoologie, 108, $739-878$. https://doi.org/10.5962/bhl.part.80165

Tamura, K., Stecher, G., Peterson, D., Filipski, A. \& Kumar, S. (2013) MEGA6: Molecular Evolutionary Genetics Analysis version 6.0. Molecular Biology and Evolution, 30, 2725-2729.

Torrey, H.B. (1909) The Leptomedusae of the San Diego region. University of California Publications in Zoology, 6 (2), $11-31$.

Uchida, T. (1933) Medusae from the vicinity of Kamchatka. Journal of the Faculty of Science, Imperial University of Tokyo, Zoology, 2 (3), 125-133.

Xu, Z.Z. \& Huang, J.Q. (2006) On new genus, species and record of Laingiomedusae and Anthomedusae in Fujian coast (Cnidaria, Hydroidomedusae). Journal of Xiamen University (Natural Science), 45 (2), 233-249. 